\title{
Publisher's Note: Nonradiative limitations to plasmon propagation in chains of metallic nanoparticles [Phys. Rev. B 94, 205432 (2016)]
}

Adam Brandstetter-Kunc, Guillaume Weick, Charles A. Downing, Dietmar Weinmann, and Rodolfo A. Jalabert (Received 2 December 2016; published 13 December 2016)

DOI: 10.1103/PhysRevB.94.239902

This paper was published online on 28 November 2016 with an error in Eq. (B13). Equation (B13) should read as

$$
\gamma_{ \pm}^{\sigma, \mathrm{r}}=\frac{2 \omega_{ \pm}^{\sigma 3}}{3 c^{3}}\left(\sum_{n=1}^{2} \sqrt{\omega_{n} a_{n}^{3}} \Delta u_{n \pm}^{\sigma}\right)^{2}
$$

The equation has been corrected as of 2 December 2016. The equation is correct in the printed version of the journal. 